\title{
The Philosophical Thinking on the Combination between Traditional Chinese and Western Medicine to the Hearing Loss Relating to Diabetes
}

\author{
Ruiyu $\mathrm{Li}^{1, \mathrm{a}}$, Xueyu Ma ${ }^{2, \mathrm{~b}}$, Mishan $\mathrm{Wu}^{3, \mathrm{c}}$, Junqiao Guo ${ }^{1, \mathrm{a}}$, Meng $\mathrm{Li}^{4, \mathrm{~d}}$, Yue $\mathrm{Li}^{5}$, \\ e, Ying Dang ${ }^{6, f}$ and Weihua Han ${ }^{1, a}$ \\ ${ }^{1}$ Institute for Integrated Traditional and Western Medicine, Second Affiliated Hospital of Xingtai \\ Medical College, Xingtai, 054000, China \\ ${ }^{2}$ Xingtai University, Department of Education Science Teaching, Xingtai, 054000, China \\ ${ }^{3}$ Department of Prescription, Hebei College of Traditional Chinese Medicine, Shijiazhuang, 050090, \\ Hebei Province, China \\ ${ }^{4}$ Health Team, Hotan Detachment of the Xinjiang Armed Police Corps,Hotan, 848011, Xinjiang \\ Uygur Autonomous Region, China \\ ${ }^{5}$ Department of psychology, Chengde Medical College, Chengde, 054000, China \\ ${ }^{6}$ Department of Clinical Surgery, Xingtai Medical College, Xingtai 054000, Hebei Province, China \\ Corresponding author: Li Ruiyu \\ aemail: Liruiyu651021@163
}

\begin{abstract}
Keywords: Combination of TCM and western medicine; Diabetes; Hearing loss; Philosophy; Dialectical materialism
\end{abstract}

Abstract. The basic theories of TCM have the same philosophy with Taoism, Confucianism and other traditional Oriental ideology. TCM holds that all the natural phenomena have two aspects, Yin and Yang, which are both contradictory and complement to each other, as well as interdependence and mutual transformation. From the relation between the "kidney deficiency" of TCM and osteocalcin of modern research, this thesis aims to explore the dynamic equilibrium relationship maybe existed in diabetes with "kidney deficiency" and "kidney dominating bones", reinforcing kidney with osteoblast, osteoclast, osteocalcin and insulin, including simple philosophy of traditional Chinese medicine knowledge, the relationship between kidney deficiency and diabetes, the philosophical thinking on the hearing loss relating to diabetes, the philosophical thinking on the effect of bone metabolism to diabetes. In the part of conclusion, from the perspective of dialectical materialism and the simple philosophy of traditional Chinese medicine, this thesis simplify complex problems, based on theory of Yin and Yang, and from the correlation between "kidney advocate bone" contacting "kidney deficiency " as well as osteocalcin and diabetes, seizing the main contradiction, viewing the illness development from philosophy, and thus this thesis probably has a breakthrough in the hearing loss and other diabetic complications in diabetes, as well as diabetes research and prevention.

\section{Introduction}

Diabetes is a kind of chronic endocrine metabolic disease caused by multiple factors, due to glucose metabolic disorder leading to high blood sugar and then a series of physiological function disorder diseases caused by protein, fat, and electrolyte. Traditional Chinese medicine syndrome differentiation classified "excessive" conditions, to drink, eat, diuresis and hand-picked as its typical performance, and it has a genetic predisposition. Diabetes is a chronic progressive disease. There is still no ideal treatment. Hearing loss is one of diabetic complications, and its mechanism has not yet fully understood. The main reason is that occurrence of diabetes has genetic polymorphism. TCM holds that diabetes has close relationship with "kidney deficiency". Lee and other researchers found that there was close relationship between diabetes and osteocalcin. And the TCM reinforcing kidney has improvement and regulation function to osteoblast and diabetes patients with deafness and bone calcium. TCM holds that there may be a dynamic equilibrium relationship in "kidney deficiency" and 
"kidney advocate bone", reinforcing kidney with osteoblast, osteoclast, osteocalcin and insulin. This paper preliminary has the following thinking from philosophy.

\section{Philosophical Thinking on Diabetic kidney Deficiency and Kidney Advocating Bone, Osteoblast and Osteocalcin Secreting Insulin}

Knowledge on the Naive Philosophy of TCM. The basic theories of TCM have the same philosophy with Taoism, Confucianism and other traditional Oriental ideology. TCM holds that all the natural phenomena have two aspects, Yin and Yang, which are both contradictory and complement to each other, as well as interdependence and mutual transformation. Everything in the universe is made up of wood, fire, earth, metal and water of five basic elements. Everything is in permanent change, and gains the dynamic balance and harmony in the changes. These naive philosophies are used to explain the disease mechanism and to find the strategy of prevention and treatment of disease, and formed early basic thought of traditional Chinese medicine. Yin and Yang are against each other, mutual restriction and mutual exclusion, and achieve the relative dynamic balance to its unified. Engels said: "In a living organism, we see all the smallest part and larger organs continuing movement, the movement in the normal period of life takes the sustainable balance of the body as the result, and often is in the movement. This is the unity of the movement and cooperation." Yin and Yang adjust each other and maintain the relative balance, which is the basic condition for the normal life activities.

The Relation with Kidney Deficiency and Diabetes. In TCM, diabetes was called "emaciation thirst", and "emaciation thirst" closely related to the kidney deficiency, which means the body loss balance of Yin and Yang and performs the kidney deficiency. There are many records on it. Emaciation-thirst Disease is easy to viscera, and kidney deficiency can deafness, blindness, cataracts and so on. In China, Zhang Hui found the "lumbar debility of diabetes has close relationship with kidney". Tan[8] researches "the relation between kidney deficiency and family heredity of diabetes", "the distribution characteristics of kidney deficiency as well as a research to gene expression profiles and diabetic pedigree", which illustrate the close relationship with diabetes From the angle of kidney deficiency. Generally, there is relation between the diabetes and kidney deficiency. By means of drug test, it found that the rat with kidney deficiency has a bone metabolism disorder of two gene regulation line, nerve-endocrine-immune and nerve-endocrine-bone metabolism. Reinforcing kidney can correct this network function[9].

The Philosophy to the Hearing Loss of Diabetes. Dialectical materialism thought that all things in the world is something internal contradictory relationship between the two sides, both opposite and unified things change and development. The Hearing Loss of Diabetes, the first should be "emaciation-thirst", and then hearing Loss, kidney deficiency is fundamental, and the hearing Loss is just the sign, because diabetes importantly lies on the kidney deficiency. At present, the occurrence of diabetes complicated with hearing loss nature is neither the concept of point, nor the line, its performance for the multi-level and multi-channel is complex network system. Dialectical materialism thought that constituting things lie in all aspects of a variety of contradictions, which requests us to unify materialist dialectics and Key Theory together. We should find out the various aspects of things, and distinguish between primary and secondary, seize the main contradiction and conflict. Because the nature of things is mainly depended by the key aspect of principal contradiction, we can't reverse the primary and secondary. Lee found that there was close relationship between diabetes and osteocalcin, and TCM to reinforce kidney can improve osteoblast and osteocalcin of diabetes patients with deafness. According to the theories on "kidney dominating bones" and "kidney opening at ear", the author puts forth the scientific hypothesis on the integrative theory between reinforcing kidney and "diabetes- kidney-bone-ear". (diabetes with hearing loss by kidney deficiency $\rightarrow$ reinforcing kidney $\rightarrow$ osteoblast $\rightarrow$ osteocalcin $\rightarrow$ reinforcing the expression of insulin in islet cells $\rightarrow$ hypoglycemic effect $\rightarrow$ improving hearing). With the guidance of TCM, the author analyzes the relationship between "kidney" and "osteocalcin", and highlight the advantages and characteristics on cooperation of Chinese and western medicine. Therefore, diabetes complicated 
with hearing loss should depend on the principal contradiction, disorder of Yin and Yang on kidney deficiency, then leading to influence the insulin secretion by osteoblast, osteoclast, and osteocalcin. Secretion shortage of insulin can aggravate the imbalance of Yin and Yang.

\section{Philosophy on the Effect of Bone Metabolism to Diabetes}

Hearing loss caused by diabetes is mainly because of secretion shortage of insulin, and leads to the imbalance of osteoblast, osteocalcin and insulin. According to the theory of "kidney advocate bone", the author regards bone metabolism formed by the "kidney deficiency" as the main contradiction.

Normal bone metabolism: normal bone metabolism in body is a complex process in which the bone tissue continues to renovate, including two aspects of bone resorption and bone formation. Generally, this process order: activation $\rightarrow$ bone resorption $\rightarrow$ bone formation. Firstly, osteoclasts involved in bone resorption are activated, which dissolve matrix, remove the calcium in bone out and reinforce the bone resorption. Then, there are forming the osteocyte in the surface of the bone resorption. Osteoblasts synthesize bone matrix of non-mineralization, and send the calcium to calcification zone. Finally, crystallization of calcium and phosphores gradually deposit in bone matrix, and then calcification of bone matrix forms the bone tissue. In the process of bone metabolism, a certain amount of bone tissue is absorbed daily, and certain amount of bone tissue is formed. Both of them are balanced, which plays an important role in maintaining the bone mineral density [10]. Reconfiguration process of skeletal system penetrates the whole life, and the whole process is regulated by the partial or entire factors [11-13].

Gopalakrishnan reports that high glucose $(16.5 \mathrm{mmol} / \mathrm{L}$ and $49.5 \mathrm{mmol} / \mathrm{L})$ can show the concentration dependent to inhibit mesenchymal cells in bone marrow to differentiate into osteoblasts. The studies of Zhen show that osteoblasts derived from the skull are inhibited to differentiate in the high glucose environment. Under high glucose ( $22 \mathrm{mmol} / \mathrm{L}$ and $44 \mathrm{mmol} / \mathrm{L})$, calcium nodules formed by osteoblasts are not only less number than control group, but also the number of calcium deposition of single calcium nodules is less. High glucose ( $44 \mathrm{mmol} / \mathrm{L})$ would obviously inhabit gene expression of Runx2, IGF-1 and IGF-1R in osteoblasts. All these imply that the effect of high glucose to osteoblasts is by influence the expression of Runx2, IGF-1 and IGF-1R. García-Hernández thought that the high glucose in extracellular fluid can change the mineralization function of osteoblasts. Meanwhile, Freude indicated that the environment with insulin would have the damage to osteoblasts, but they also thought that the reason that high glucose and insulin can increase the differentiation of osteoblasts may be due to the increasing of energy equilibrium [17-18]. Through the insulin signaling pathway, osteoblasts exposed to high insulin environment can increase the synthesis of collagen, the production of alkaline phosphatase and glucose absorption, which increase osteoblast differentiation and strengthen the function of osteoblast [19-21].

Kidney deficiency affects "kidney dominating bones" function, while "kidney advocate bone" function is reduced, which can cause the damage to bone metabolism of type 2 diabetes, lower bone transformation, and maybe inhabit the formation of osteoblasts as well as the resorption of osteoclast. But the inhibitory effect on bone formation is stronger. Given the gene research observation on the osteoblast expression in mice by Lee, the author found that the osteocalcin is a kind of protein that is regulated by the ESP. And ESP defect promotes the beta cell proliferation in mice, and increases insulin secretion. Osteocalcin can improve the level of insulin secretion, insulin sensitivity, and inhabit fat accumulation. Therefore, kidney deficiency would affect bone metabolism, and its abnormalities have an influence on the balance of osteoblast and osteoclast, which also would cause the damage to insulin secretion and its decline leads to hyperglycaemia. At last, the hyperglycaemia damage to bone metabolism.

\section{Conclusion}

Thinking is more important than collecting information, especially the philosophical thinking. Medicine is relatively slow progress in natural science (if not the slowest) subjects. The structure and 
function of human body as well as the interactions with drugs are so complex, and even in the contemporary the most advanced science and technology will be overshadowed by in front of it. The right conclusion, sometimes even still depends on ancient naive materialism view of traditional Chinese medicine to confirm. There is no limit to the number of the unknown, and the known is limited. On the way of exploring the unknown, confusion is not always a rushed traveler, instead it may be our partner at hand.

Dialectical materialism thinks that things are moving, changing and developing. We should view and understand the problem from the dynamic, developing, changeable aspect. In contemporary internet era, everything is related, mutual influence, and mutual balance, which provides a powerful ideological basis to find and solve problems. In terms of diabetes complicated with hearing loss, it is caused by a complex, multiple factors, more balance disorders system. At present, medical scientists all over the world in the prevention and control of diabetes research has done a lot of work, but very little breakthrough it has achieved. From the point of view of dialectical materialism and naïve philosophy of traditional Chinese medicine, we simplify complex problems. Based on theory of Yin and Yang, by "kidney advocate bone" relating "kidney deficiency" as well as the relation between osteocalcin and diabetes, we should seize the main contradiction, and view the illness development philosophically, which probably have a breakthrough on diabetes and diabetes with hearing loss as well as other complications in diabetes and prevention.

\section{References}

[1] Lee N K, Sowa H and Hinoi E: Cell. Vol. 130 (2007). p. 456-469

[2] Wei Shuangping, Li Meng and Li Ruiyu: Tissue engineering research in China Vol. 17(2013). p. 2003-2090

[3] Li Ruiyu, Li Meng and Guo Ynling: New TCM Vol. 43 (2011). p. 32-33

[4] Chen KJ and Xu H: European Rev Vol. 2003, 11 (2003). p. 225-235

[5] Kaptchuk TJ: The Web That Has No Weaver: Understanding Chinese Medicine (McGraw-Hill Education, Illinois 2000).

[6] Zhang Hui, Ni Qing and Ren Yan: TCM Journal Vol. 51 (2010). p. 409-411

[7] Zhang Hui,Wang Jingrong and Tan Conge: TCM Journal in Liaoning Vol. 37(2010). p. 1023-1025

[8] Tan Conge, Wang Miqu and Ni Qing: TCM Journal in Liaoning Vol. 37(2010). p. 1197-1199

[9] Shen Ziyin, Chen Yu and Huang Jianhua: Journal on the Combination of TCM and Western Medicine in China Vol. 26 (2006). p. 521-525

[10]Raisz LG: J ClinInvest Vol. 115(2005). p. 3318-3325

[11]Boyle WJ, Simonet WS and Lacey DL: Nature Vol. 423(2003). p. 337-342

[12] Rauner M, Sipos W and Pietschmann P: Int Arch Allergy Immunol Vol. 143(2007). p. 31-48

[13] Sipos W, Pietschmann P and Rauner M: Wien Med Wochenschr Vol. 159(2009). p. 230-234

[14] Gopalakrishnan V, Vignesh RC and Arunakaran J: Biochem Cell Biol Vol. 84(2006). p. 93-101

[15]Zhen D, Chen Y and Tang X: J Diabetes Complications Vol. 24(2010). p. 334-344

[16] Garcia-Hernandez A, Arzate H and Gil-Chavarria I: Bone Vol. 50(2012). p. 276-288

[17]Freude T, Braun KF and Haug A: J Mol Med (Berl) Vol. 90(2012). p. 1257-1266

[18]Ferron M, Wei J and Yoshizawa T: Cell, 142(2010). p. 296-308 
[19]Kream BE, Smith MD and Canalis E: Endocrinology Vol. 116(1985). p. 296-302

[20]Pun KK, Lau P and Ho PW: J Bone Miner Res Vol. 4(1989). p. 853-862

[21] Ituarte EA, Halstead LR and Iida-Klein A: Calcif Tissue Int Vol. 45(1989). p. 27-33 